\title{
Populations of massive stars in galaxies, implications for the stellar evolution theory
}

\author{
Georges Meynet ${ }^{1}$, Patrick Eggenberger ${ }^{2}$ \\ and André Maeder ${ }^{1}$ \\ ${ }^{1}$ Geneva Observatory, University of Geneva, CH-1290 Sauverny, Switzerland \\ email: georges.meynet@obs.unige.ch; Andre.Maeder@obs.unige.ch \\ ${ }^{2}$ Liège University, AGO Department, B-4000 Liège 1 \\ email: eggenberger@astro.ulg.ac.be
}

\begin{abstract}
After a brief review of the observational evidences indicating how the populations of Be stars, red/blue supergiants, Wolf-Rayet stars vary as a function of metallicity, we discuss the implications of these observed trend for our understanding of the massive star evolution. We show how the inclusion of the effects of rotation in stellar models improves significantly the correspondence between theory and observation.
\end{abstract}

Keywords. stars: evolution, rotation, early-type, supergiants, Wolf-Rayet

\section{Massive star populations and stellar models}

For constraining stellar models, the best way is to compare the results of tailored theoretical models with well observed characteristics of single stars. The case of the Sun is in that respect exemplar. Considering populations of massive stars for constraining stellar models may appear at first sight a loose way to proceed since in addition to the physics of stars, other ingredients enter into the comparison as the Initial Mass Function (IMF) and the Star Formation History. However in some circumstances, observed stellar populations may provide powerful constraints. For instance, IMF has no influence on ratios of massive stars involving stars of about the same range of initial masses (as for instance ratios of blue to red supergiants, of Wolf-Rayet (WR) to O-type stars). Star formation history is not involved either provided we concentrate on regions of constant star formation (i.e. constant during at least the last 10-20 millions years). In that case the number ratios of massive stars in two different evolutionary stages is given by the ratio of the durations of these evolutionary stagest. In the following we briefly recall the main characteristics of the observed populations of Be stars, red and blue supergiants and Wolf-Rayet stars and deduce some consequences for the stellar models. The main conclusion is that, in all cases, rotation appears as a key physical ingredient in order to reproduce the mean features of these populations. We end by saying a few words about the consequences for nucleosynthesis, and again we confirm that rotation plays a key role in this area also. Before addressing these questions, let us say a few words about the effects of rotation.

$\dagger$ Let us note that for starburst the situations is different, because the massive star population varies as a function of the age and depends also on the duration and intensity of the initial burst of star formation. 


\section{Rotating models}

The physics of rotation has been extensively discussed in previous papers (see e.g. Zahn 1992; Maeder \& Meynet 2001) to which the reader can refer. Massive star grids of nonrotating and rotating models have been made at $Z=0.040,0.020,0.008,0.004$ and $10^{-5}$ (Meynet \& Maeder 2005). The rotating models have an initial velocity $v_{\text {ini }}$ of $300 \mathrm{~km} / \mathrm{s}$, which gives an average velocity of $220 \mathrm{~km} / \mathrm{s}$ during the MS phase, corresponding to observations. The main effects of rotation are the following:

- Rotation increases the MS lifetime with respect to non-rotating models (up to about $40 \%$ for the most massive stars and for very fast rotation).

- The values assigned from isochrones with an average rotation velocity typically lead to ages $25 \%$ larger than without rotation.

- These models account for the observed changes of the surface abundances for OB main sequence and supergiants stars (see also Heger \& Langer 2000).

- Steeper gradients of internal rotation $\Omega$ are built at lower $Z$. The steeper $\Omega$-gradient at lower $Z$ favors mixing. There are 2 reasons for the steeper $\Omega$-gradients. One is the higher compactness of the star at lower $Z$. The second one is that at lower $Z$, the density of the outer layers is higher, thus the meridional currents are slowlyer. This produces less outward transport of angular momentum and contributes to steepen the $\Omega$-gradient.

- At lower $Z$, rotating stars more easily reach break-up velocities and may stay at break-up for a substantial fraction of the MS phase.

The different scenarios for the evolution of massive stars are indicated below.

$M>90 M_{\odot}:$ O - Of - WNL - (WNE) - WCL - WCE - SN (Hypernova low Z ?)

$\overline{60-90 M_{\odot}}: \mathrm{O}-\mathrm{Of} / \mathrm{WNL} \Leftrightarrow \mathrm{LBV}-\mathrm{WNL}(\mathrm{H}$ poor $)-\mathrm{WCL}-\mathrm{E}-\mathrm{SN}(\mathrm{SNIIn} ?)$

$\overline{40-60 M_{\odot}}: \mathrm{O}-\mathrm{BSG}-\mathrm{LBV} \Leftrightarrow \mathrm{WNL}-(\mathrm{WNE})-\mathrm{WCL}-\mathrm{E}-\mathrm{SN}(\mathrm{SNIb})$

- WCL-E - WO SN (SNIc)

$30-40 M_{\odot}: \mathrm{O}-\mathrm{BSG}-\mathrm{RSG}-\mathrm{WNE}-\mathrm{WCE}-\mathrm{SN}(\mathrm{SNIb})$

$\mathrm{OH} / \mathrm{IR} \Leftrightarrow \mathrm{LBV}$ ?

$25-30 M_{\odot}:$ O -(BSG)- RSG - BSG (blue loop) - RSG - SN(SNIIb, SNIIL)

$\overline{10-25 M_{\odot}}:$ O - RSG - (Cepheid loop, $\left.M<15 M_{\odot}\right)$ RSG - SN (SNIIL, SNIIp)

The sign $\Leftrightarrow$ means back and forth motions between the two stages. The limits between the various scenarios depend on metallicity $Z$ and rotation. The various types of supernovae are tentatively indicated.

\section{The Be stars}

Be stars are emission line stars. Emission originates in a circumstellar outflowing disk. How do these disks form? How long are their lifetimes? Are they intermittent? Are they Keplerian? Many of these questions are still subject of lively debate. A point however which seems well accepted is the fact that the origin of a disk might be connected to the fast rotation of the star (Pelupessy et al. 2000). Martayan et al. (2006) showed that the initial velocities of the Be stars is significantly higher than the initial velocities of the normal B stars, giving some support to the view that only stars with a sufficiently high initial velocity can go through a Be star episode.

The population of Be stars varies with the metallicity. Fig. 1, left panel, shows that the number of Be stars with respect to the total number of B stars (B and Be stars) in cluster with ages between 10 and $25 \mathrm{My}$ (mass at the turn off between about 9 and $15 \mathrm{M}_{\odot}$ ) increases with decreasing metallicity (Maeder et al. 1999). Such a trend has been recently confirmed by Wisniewski \& Bjorkman (2006). Very interestingly there appears 

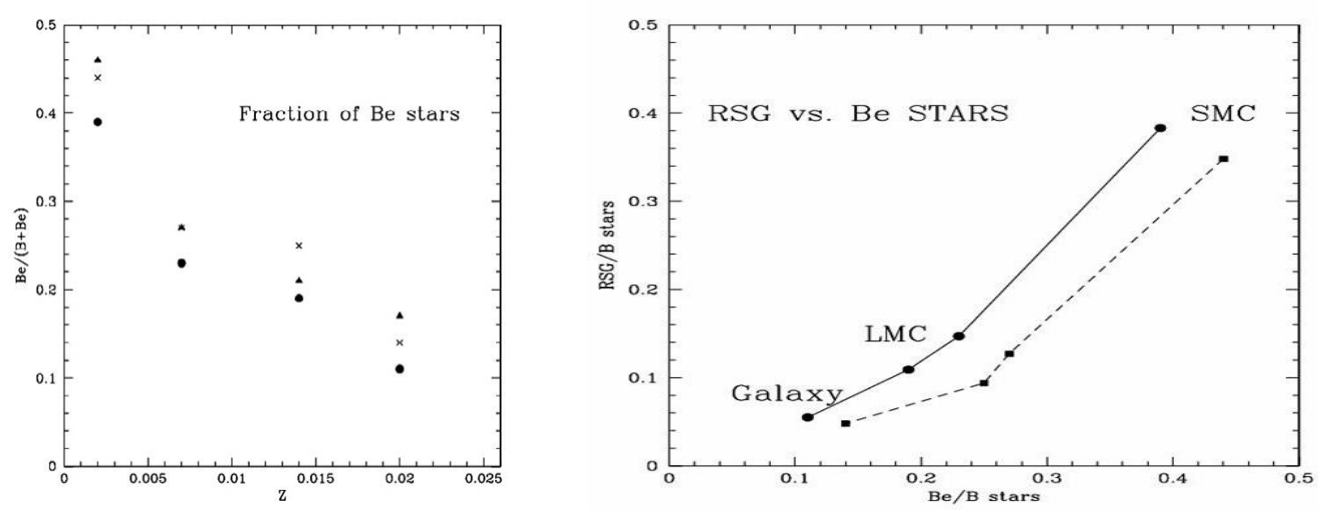

Figure 1. Left panel: Variation as a function of metallicity of the number ratio of Be stars to $\mathrm{B}$ and Be stars (Maeder et al. 1999) Right panel: Correlation between the the number of red supergiants and the number ratio of Be to B stars.

to be a correlation between the frequency of Be stars and that of red supergiants as shown in Fig. 1 right panel.

These observations indicate that metallicity plays a role in the Be phenomenon and provides hints on the way surface velocity may evolve differently for stars of different initial metallicities. The correlation of Be star populations with those of red supergiants can be seen as an indication that fast rotation not only favors the formation of Be stars but also of that red supergiants.

As long as the mass losses by stellar winds are not too strong (which is the case for either low metallicity and/or initial masses below about $15 \mathrm{M}_{\odot}$ ), the surface equatorial velocity increases with time during the MS phase. This is due to the coupling between the core and the envelope exerted by the meridional currents. These currents tends to slow down the contracting, still faster spinning core, and to accelerate the expanding outer envelope. Due to this effect, a star beginning its evolution on the ZAMS with a sufficiently high initial rotation rate, may reach the break-up limit at a given point during the MS phase (the break-up limit is the point at which the equatorial velocity is such that the centrifugal acceleration is equal to the gravity at the equator).

In Fig. 2, the lifetimes on the MS of different $9 \mathrm{M}_{\odot}$ stellar models corresponding to various initial values of $\Omega / \Omega_{\text {crit }}$ are shown as well as the time at which the surface equatorial velocity reaches the critical limit. On can see that

- Only those stars, beginning with an initial angular velocity on the ZAMS such that it corresponds to about $70 \%$ of the critical angular velocity will reach the critical limit during the MS phase.

- The model starting with $\Omega / \Omega_{\text {crit }}=0.99$ reaches the critical limit only after an age of 22 millions years. This comes from the fact that, at the very beginning of the MS phase, meridional currents transport angular momentum from the outer part of the star to the inner one, accelerating the core and slowing down the envelope. Thus the star first evolves away from the critical limit. This phase is quite short lasting only a few percents of the MS lifetime. Then the meridional currents reverse in the outer layers making the surface velocity to approach the critical limit.

- Those stars which reach the critical limit, do it at the end of the MS phase.

If we accept the premise that a Be star is a star near the break-up limit, then Be stars might naturally arise from the normal evolution of stars beginning their life with a sufficiently high velocity. The question is of course to know if such a scenario might 


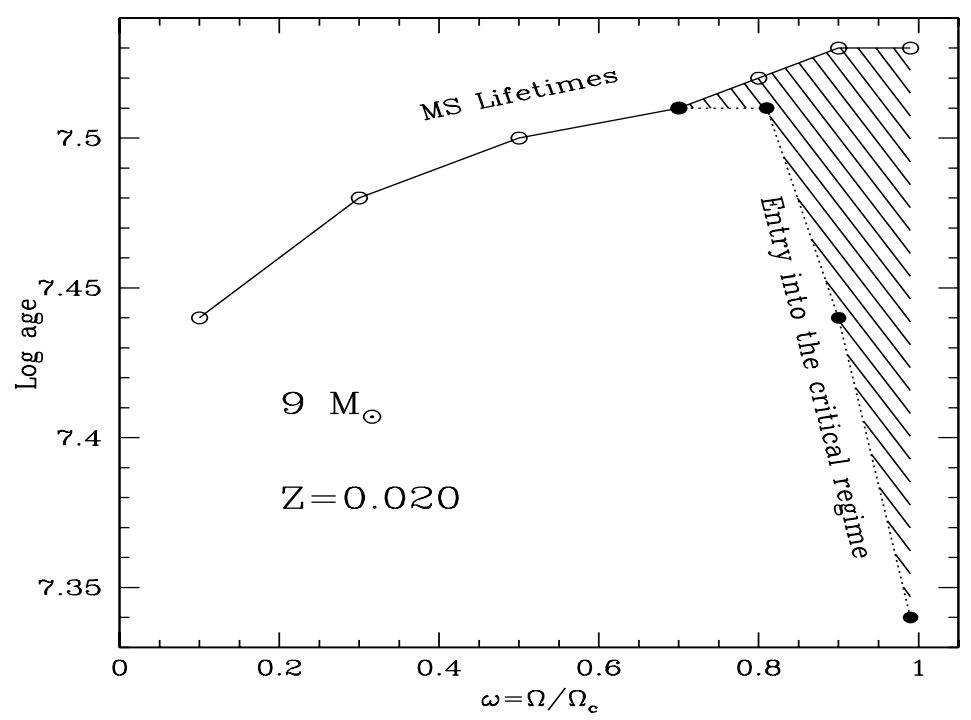

Figure 2. The MS lifetime of $\mathrm{Z}=0.029 \mathrm{M}_{\odot}$ is plotted for different initial velocities (continuous line with empty circles). The age at which the star encounters for the first time the critical limit is indicated (dotted line with full circles). The hatched zone shows the region where stars at the critical limit are expected.

account for the observed ratios and their observed variation with the metallicity (see Fig. 1). While these points remain to be carefully examined, one can already say that from a theoretical point of view, one would expect a higher proportion of Be stars at low $\mathrm{Z}$ if the distribution of the initial velocity is biased toward faster rotators at low $Z$ and/or if, due to the weaker stellar winds at low metallicity, less angular momentum is removed from the surface.

\section{The supergiants}

In Fig. 3, left panel, is shown the variation with the metallicity of the number ratio of blue to red supergiants $(\mathrm{B} / \mathrm{R})$, and (right panel) its variation as a function of the galactocentric distance in the Milky Way (Meylan \& Maeder 1982; Eggenberger et al. 2002). Clearly, the $B / R$ ratio increases when the metallicity increases, while standard stellar evolution models (i.e. models without any extra-mixing processes) predict that this ratio should decrease with increasing metallicity. Dohm-Palmer \& Skillman (2002) have estimated the $\mathrm{B} / \mathrm{R}$ ratio in the dwarf irregular galaxy Sextans $\mathrm{A}$ for various age bins spanning a range between 20 and $140 \mathrm{My}$. They find that the observed $\mathrm{B} / \mathrm{R}$ are lower than those given by standard models by a factor 2 , indicating that too few red supergiants are predicted by these models.

This disagreement implies that no reliable predictions can be made concerning the nature of the supernova progenitors in different environments, or the populations of supergiants in galaxies. The $\mathrm{B} / \mathrm{R}$ ratio also constitutes an important and sensitive test for stellar evolution models, because it is very sensitive to mass loss, convection and mixing processes (Langer \& Maeder 1995). Thus, the problem of the blue to red supergiant ratio $(B / R$ ratio) remains one of the most severe problems in stellar evolution.

Fig. 4, left panel, shows for the models of 15, 20 and $25 \mathrm{M}_{\odot}$ the changes of $T_{\text {eff }}$ as a function of the fractional lifetimes in the He-burning phase for different rotation. For all 

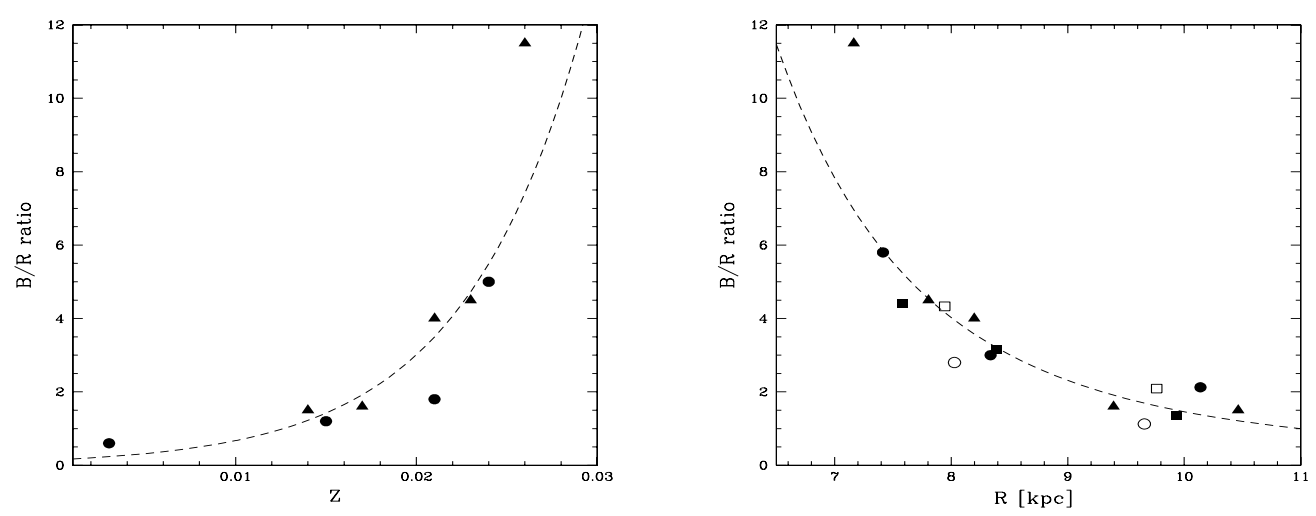

Figure 3. Left panel: $B / R$ ratio in the Galaxy and the SMC for clusters with log age between 6.8 and 7.5. The distinction between blue and red supergiants is based on spectroscopic measurements. The triangles refer to $B$ including $\mathrm{O}, \mathrm{B}$ and $\mathrm{A}$ supergiants. The dots refer to $B$ including only B supergiants. The dashed curve corresponds to the fit for $B$ including $\mathrm{O}, \mathrm{B}$ and A supergiants with $(B / R)_{\odot}=3.0$. Right panel: $B / R$ ratio in the Galaxy for different age intervals, with distinction between blue and red supergiants based on spectroscopic measurements. $B$ includes $\mathrm{O}$, B and A supergiants. The dashed curve corresponds to the same fit as in the left panel. For a detailed description of the figure see Eggenberger et al. (2002)

masses, we notice that the non-rotating stars spend nearly the whole of their He-phase as blue supergiants and almost none as red supergiants. For $v_{\text {ini }}=300 \mathrm{~km} \mathrm{~s}^{-1}$ (which corresponds to about $\bar{v}=220 \mathrm{~km} \mathrm{~s}^{-1}$ ), we notice a drastic decrease of the blue phase and a corresponding large increase of the red supergiant phase. This figures illustrates the fact that models including the effects of rotation evolves much more rapidly into the red supergiant phase than non-rotating models. Of course we have not yet the complete answer to the problem of the $\mathrm{B} / \mathrm{R}$ ratio. For this we should show that we can reproduce the plots shown in Fig. 3, but still part of the problem appears to be resolved by rotation.

The physical reason for this behavior is the following: first, let us recall that, for a given luminosity, an extended convective zone allows the star to remain more compact, the gradient of density being shallower in a convective zone than in a radiative one. In the rotating model, the convective zone associated to the H-burning shell rapidly disappears. This is mainly caused by the mild mixing just outside the core produced by rotation during the MS phase. This mixing increases the amount of helium near and above the $\mathrm{H}$-shell, and decreases the abundance of hydrogen there. This makes the H-burning shell less active (see for more details Maeder \& Meynet 2001). As a consequence the convective zone associated to it rapidly disappears making the star to evolve into the red supergiant stage. This is illustrated in Fig. 4, right panel, which shows models of a $20 \mathrm{M}_{\odot}$ star in the middle of the He-burning phase. One sees that: 1) the mixing in the MS phase leads to a slight extension of the core (this also favors a redwards motion during the He-burning phase); 2) the higher He abundance in the region of the $\mathrm{H}$-burning shell (the $\mathrm{H}$-shell is just on the right side of the big $\mathrm{He}-$ peak) which makes this region radiative (there is an abundance gradient) instead of being convective as in the non-rotating model (flat profile).

\section{The Wolf-Rayet stars}

Wolf-Rayet stars play a very important role in Astrophysics, as signatures of star formation in galaxies and starbursts, as injectors of chemical elements and of the radioactive 

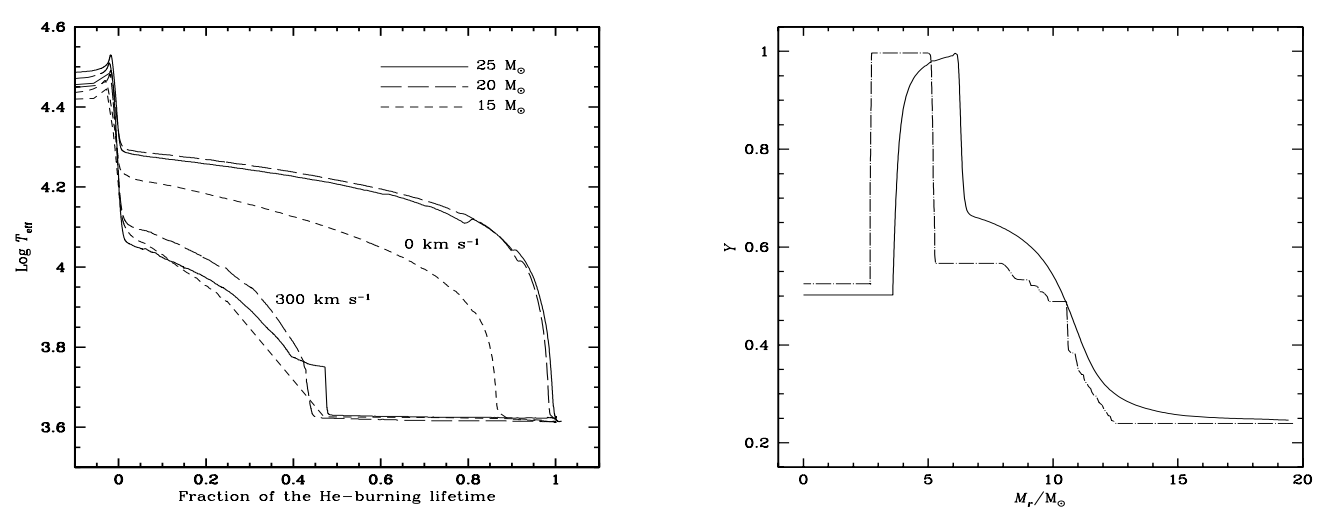

Figure 4. Left panel: Evolution of the $T_{\text {eff }}$ as a function of the fraction of the lifetime spent in the He-burning phase for 15,20 and $25 \mathrm{M}_{\odot}$ stars at $Z=0.004$ with $v_{\text {ini }}=0$ and $300 \mathrm{~km} \mathrm{~s}^{-1}$. Right panel: Comparison of the internal distribution of helium in two models of $20 \mathrm{M}_{\odot}$ at the middle of the He-burning phase. The dashed-dot line concerns the models with zero rotation and the continuous line represents the case with $v_{\text {ini }}=300 \mathrm{~km} \mathrm{~s}^{-1}$.

isotope ${ }^{26} \mathrm{Al}$, as sources of kinetic energy into the interstellar medium and as progenitors of supernovae and, likely, as progenitors of long soft $\gamma$-ray bursts.

Let us recall some difficulties faced by standard stellar models concerning the WR stars. A good agreement between the predictions of the stellar models for the WR/O number ratios and the observed ones at different metallicities in regions of constant star formation was achieved provided the mass loss rates were enhanced by about a factor of two during the MS and WNL phases (Maeder \& Meynet 1994). This solution, which at that time appeared reasonable in view of the uncertainties pertaining the mass loss rates, is no longer applicable at present. Indeed, the mass loss rates during the WR phase are reduced by a factor 2 to 3 , when account is given to the clumping effects in the wind (Nugis and Lamers 2000). Also, the mass loss rates for $\mathrm{O}$-type stars have been substantially revised (and in general reduced) by the new results of Vink et al. (2001). In this new context, it is quite clear that with these new mass loss rates the predicted numbers of WR stars by standard non-rotating models would be much too low with respect to the observations.

A second difficulty of the standard models with mass loss concerns the observed number of transition WN/WC stars. These stars show simultaneously some nitrogen characteristic of WN stars and some carbon of the further WC stage. The observed frequency of WN/WC stars among WR stars turns around 4.4\% (van der Hucht 2001), while the frequency predicted by the standard models without extra-mixing processes are lower by 1-2 orders of magnitude (Maeder \& Meynet 1994). A third difficulty of the standard models as far as WR stars were concerned was that there were relatively too many WC stars with respect to WN stars predicted (see the review by Massey 2003). These difficulties are the signs that some process is missing in standard models.

Models with rotation can account for the observed variation of the number of WR to O-type stars with the metallicity, while non-rotating models predict much too low values for these ratios when loss rates accounting for clumping effect are used (Meynet \& Maeder 2005).

Fig. 5 left shows the evolution of surface abundances for a $40 M_{\odot}$ star with and without rotation. Rotation makes smoother changes of abundances, due to internal mixing. As 

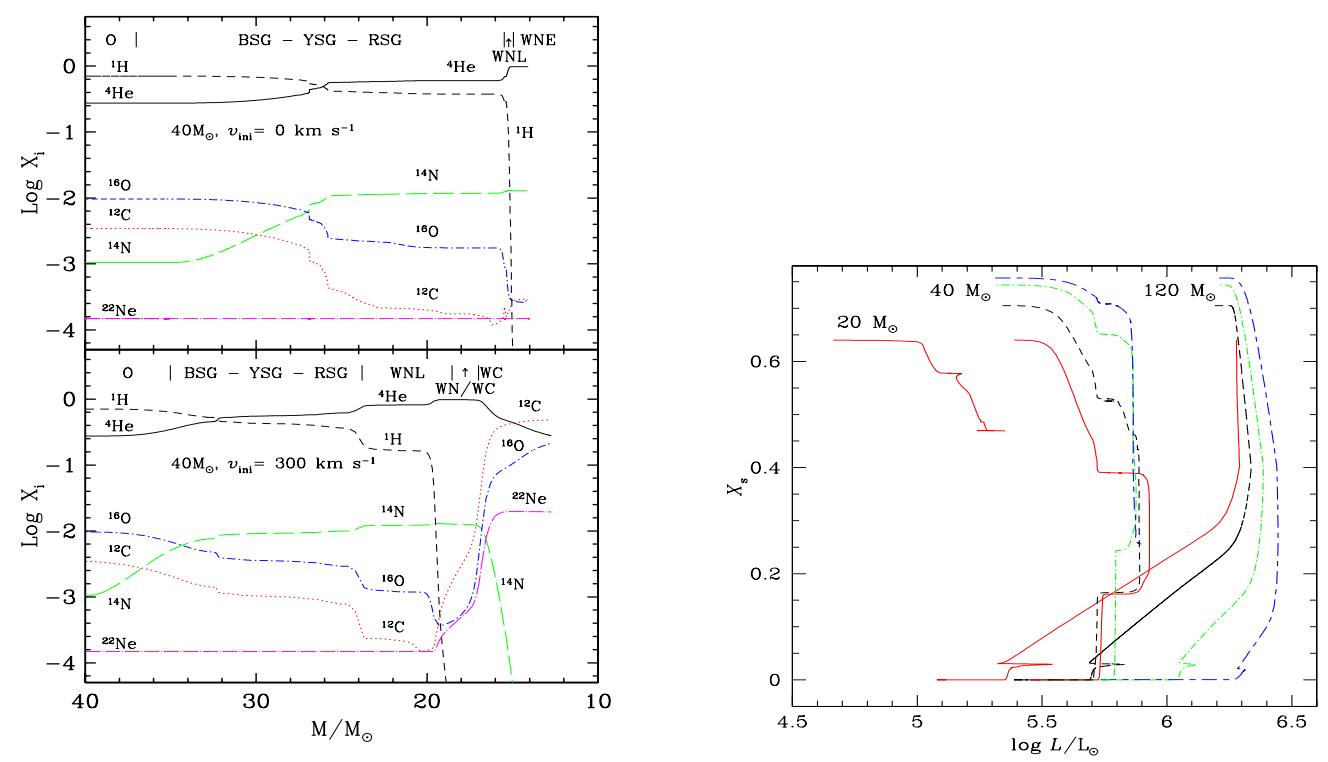

Figure 5. Left panel: Evolution as a function of the actual mass of the abundances (in mass fraction) at the surface of a non-rotating (upper panel) and a rotating (lower panel) $40 \mathrm{M}_{\odot}$ stellar model. Right panel: Evolutionary tracks in the $X_{\mathrm{s}}$ versus $\log L / L_{\odot}$ plane, where $X_{\mathrm{s}}$ is the hydrogen mass fraction at the surface. The initial masses are indicated. Long-short dashed curves show the evolution of $Z=0.004$ models, dashed-dotted curves, short-dashed curves and continuous lines show the evolutions for $Z=0.008,0.020$ and 0.040 respectively.

a consequence more stars are predicted to be in the intermediate $\mathrm{WN} / \mathrm{WC}$ phase as required by the observations.

Fig. 5 right shows the evolution of the $\mathrm{H}$-surface content $X_{\mathrm{s}}$ vs. luminosity. This is a very constraining diagram especially for the transition stages from $\mathrm{O}$, Of, LBV to WN stages. It is useful for establishing the proper filiations between such stars. It clearly supports the view that in general WN stars succeeds the LBV stage (although there may be back and forth evolution between LBV and WNL stars). We also see that descendants from high masses at higher $Z$ significantly decrease in luminosity during their evolution.

The rare WO stars, characterized by a high $\mathrm{O} / \mathrm{C}$ ratio represent a more advanced stage of nuclear processing. Curiously enough, such stars which may be the progenitors of supernovae $\mathrm{SNIb} / \mathrm{c}$ are found only at lower $Z$. The physical reasons of that have been explained by Smith \& Maeder (1991): lower $Z$ stars become WC stars (if they do it) only very late in evolution, i.e. with a high $\mathrm{O} / \mathrm{C}$ ratio. At the opposite, at higher $Z$ the WC stars may occur at an early stage of $\mathrm{He}-$ processing, i.e. with a low $\mathrm{O} / \mathrm{C}$ ratio. This is noticeable because of the possible connection WO stars - SNIb/c - GRBs ( $\gamma$ Ray Bursts). In this context, we consider WO stars as good candidate for GRB progenitors (Hirschi et al. 2005; Meynet \& Maeder 2007).

\section{Primary nitrogen production by massive stars needed at low $\mathrm{Z}$}

The observation of the N/O abundance ratio at the surface of metal poor halo star (see Spite et al. 2005) has put very interesting constraints on the nitrogen nucleosynthesis at low Z. To reproduce these observations it is necessary that massive stars produce large amount of primary nitrogen (Chiappini et al. 2005). Standard models cannot do the job 
unless some ad hoc extra mixing process is included in the stellar models. Thus again here, standard models show their limit.

Low metallicity, rotating massive stars naturally produce primary nitrogen in quantities which increase with the initial velocity (Meynet \& Maeder 2002). In order to reproduce the observations, initial rotation velocities of the order of $50 \%$ the critical velocity has to be assumed (Chiappini et al. 2006). Interestingly this may not only account for the production of primary nitrogen, but also for the upturn of the $\mathrm{C} / \mathrm{O}$ ratio observed at low $Z$ and for the scatter in the N/O ratios. Such rotations may considerably change our picture of the evolution massive stars at very low $Z$ because these stars would undergo important mass loss through various mechanisms induced by their fast rotation: many consequences are expected bearing on the origin of the C-rich extremely metal poor stars (Meynet et al. 2006), of the He-rich stars in Omega Cen (Maeder \& Meynet 2005) and of the anti correlations in the abundances of light elements at the surface of globular cluster stars (Decressin et al. 2007).

To conclude, rotation appears as a very important physical ingredients of stellar models. New grids of stellar models accounting for the effects of rotation, spanning the whole mass range of stars and all evolutionary stages are now in preparation for various metallicities. This new sets of data will hopefully provide a new tool for studying the stellar populations in galaxies.

\section{References}

Chiappini, C., Matteucci, F. \& Ballero, S.K. 2005, A\&A, 437, 429

Chiappini, C., Hirschi, R., Meynet, G., Ekstrm, S., Maeder, A., \& Matteucci, F. 2006, A\&A Letters, 449, 27

Decressin, T., Meynet, G., Charbonnel, C., Prantzos, N., \& Ekstrm, S. 2007, A\& A, in press (astro-ph/0611379)

Dohm-Palmer, R.C., \& Skillman, E.D. 2002, AJ 123, 1433

Eggenberger, P., Meynet, G. \& Maeder, A. 2002, A\& $A$ 386, 756

Heger, A., \& Langer, N. 2000 ApJ 544, 1016

Hirschi, R., Meynet, G., \& Maeder, A. $2005 A \& A 443,581$

Langer, N., \& Maeder, A. 1995, A\&SA, 295, 685

Maeder, A., \& Meynet, G. 1994, $A \mathscr{E} A$ 287, 803

Maeder, A., \& Meynet, G. 2001, A\&A 373, 555

Maeder, A., \& Meynet, G. 2005, A\& $A$ Letters, 448, 37

Maeder, A., Grebel, E.K., \& Mermilliod, J.-C. 1999, A\& $A$ 346, 459

Martayan, C., Hubert, A.-M., Floquet, M., Fabregat, J., Fremat, Y., Neiner, C., Stee, P., \& Zorec, J. 2006, A\&GA, 445, 931

Massey, P. 2003, ARAA, 41, 15

Meylan, G., \& Maeder, A. 1982, A\&A 386, 576

Meynet, G., \& Maeder, A., 2002, A\&A 390, 561

Meynet, G., \& Maeder, A., 2005, $A \mathscr{E} A$ 429, 581

Meynet, G., \& Maeder, A., 2007, $A \& A$, in press (astro-ph/0701494)

Meynet, G., Ekström, S., \& Maeder, A. 2006, A\& $A$ 447, 623

Nugis, T., \& Lamers, H. J. G. L. M. 2000, A\&A 360, 227

Pelupessy, I., Lamers, H.J.G.L.M., \& Vink, J.S. 2000, ApJ, 359, 695

Smith, L. F., \& Maeder, A. 1991 A\&A 241, 77

Spite, M., Cayrel, R., Plez, B. et al. 2005, A\&A, 430, 655

van der Hucht, K. 2001, New Astron. Reviews, 45, 135

Vink, J.S., de Koter, A., \& Lamers, H.J.G.L.M. 2001, A\&A, 369, 574

Wisniewski, J.P., \& Bjorkman, K.S. 2006, ApJ 652, 458

Zahn, J.-P. 1992, A\&A 265, 115 


\section{Discussion}

BECKMAN: You use rotation at low metallicity to increase the fraction of WR stars by bringing up elements to the surface. Are these WR stars the same in term of temperature as the WR stars which have enhanced surface temperatures due to mass loss.

MEYnet: A star is supposed to become a WR when it is hot enough (typically $\log \mathrm{T}_{\text {eff }}>$ 4.0) and has a mass fraction of $\mathrm{H}$ at the surface inferior to 0.4. The same criterion is applied for rotating and non-rotating models.

BLum: A comment: you mentioned that here are not clusters observed in which we see RSG and WR stars. But the Galactic Center clusters do show both types of stars (Quintuplet cluster, central cluster).

Meynet: Thanks for this information. To my knowledge these are the only clusters with maybe Westerlund 1, which present such a characteristic. This may indicate that at high metallicity (at least for galactic center clusters) high mass loss rates during the RSG stage allow some stars to evolve from the RSG phase to the WR one.

WEISs: Do you take into account the coupling between chemical composition at the surface and mass loss via the composition dependence of opacities which therefore influence the effective temperature?

MEYNET: During the MS phase we do not account for this effect although when the star is in rotation the $\mathrm{C} / \mathrm{N}$ ratio decreases. During the $\mathrm{WR}$ phase the rates given by Nugis \& Lamers (2000) gives a dependance of the mass loss rates with the surface abundance. In addition to that, Vink et al. (2006) obtained on the basis of theoretical models of winds that the winds of WR stars are, as those of O-type stars, metallicity dependent.

CAssisi: How does the existence in the H-R of regions where mass loss seems to be enhanced (see Vink et al.'s papers), affect your scenario?

Meynet: )We use in our models the rates of mass loss by Vink et al. (2000;2001). For O-type stars on the MS recent mass loss rate determination by UV resonance lines seem rather to indicate lower than previously thought mass loss rates. This poses indeed a problem since it would be difficult to decrease the mass loss rates by more than an order of mangnitude to understand how Wolf-Rayet stars form unless mixing plays a dominant role in the scenario leading to Wolf-Rayet stars or stars lose a lot of mass during the Luminous Blue Variable phase.

Peterson: Would you like to comment on the possibility that the first generation stars have a different mass function than do the later generations?

Meynet: Common wisdom indeed believe that Population III stars had much higher masses than stars formed in the present Universe. The absence of dust makes cooling processes much less efficient and allowed the fragmentation process to stop early when the first stars formed. These are good theoretical reasons for believing that more massive stasr were formed. However these massive stars should contribute also to the early chemical enrichment of the ISM when they explode as pair instability supernovae, which have specific nucleosynthetic signatures: strong odd-even effects, low Zinc abundance. These features are presently not observed at the surface of extremely metal poor stars. This may be due either to the fact that these stars did not actually formed, or if formed 
had a different evolution preventing them from exploding as pair instability supernovaes, or they formed, exploded as pair instability supernovae, but still not sufficiently low metallicity stars have been observed to see their signatures.

CERviÑo: About evolutionary tracks with rotation: 1) will be there different sets with different rotational velocities?. It wil be needed to add an additional distribution (the one with initial rotation velocities) to population synthesis models. 2) Rotation produuces a deformation in the star, hence the tracks and isochrones will not be single lines but bands in the HR diagram, since temperature varies along the star and depends on the inclination angle. How would it affect population synthesis results?

MEYNET: 1) We will produce tracks for $v_{r o t}=0$ and for initial rotations corresponding to $\Omega / \Omega_{c}=0.4$ on the ZAMS for stars more massive than $\sim 1.5 \mathrm{M}_{\odot}$. For lower mass stars lower initial rotation rates are considered. The rotating tracks will have average rotational velocities during the MS phase well in the observed range, thus the rotating tracks are expected to reproduce well the average case. Of course for a few initial masses, we shall produce tracks for a larger range of rotational velocities. 2) We shall provide simple numerical code which will allow to reconstruct the evolutionary tracks for different inclination angles. The tables to be published will give, among many other quantities, the total bolometric luminosity and a mean effective temperature defined by $L=S T_{\text {eff }}$, where $\mathrm{S}$ is the surface of the deformed star.

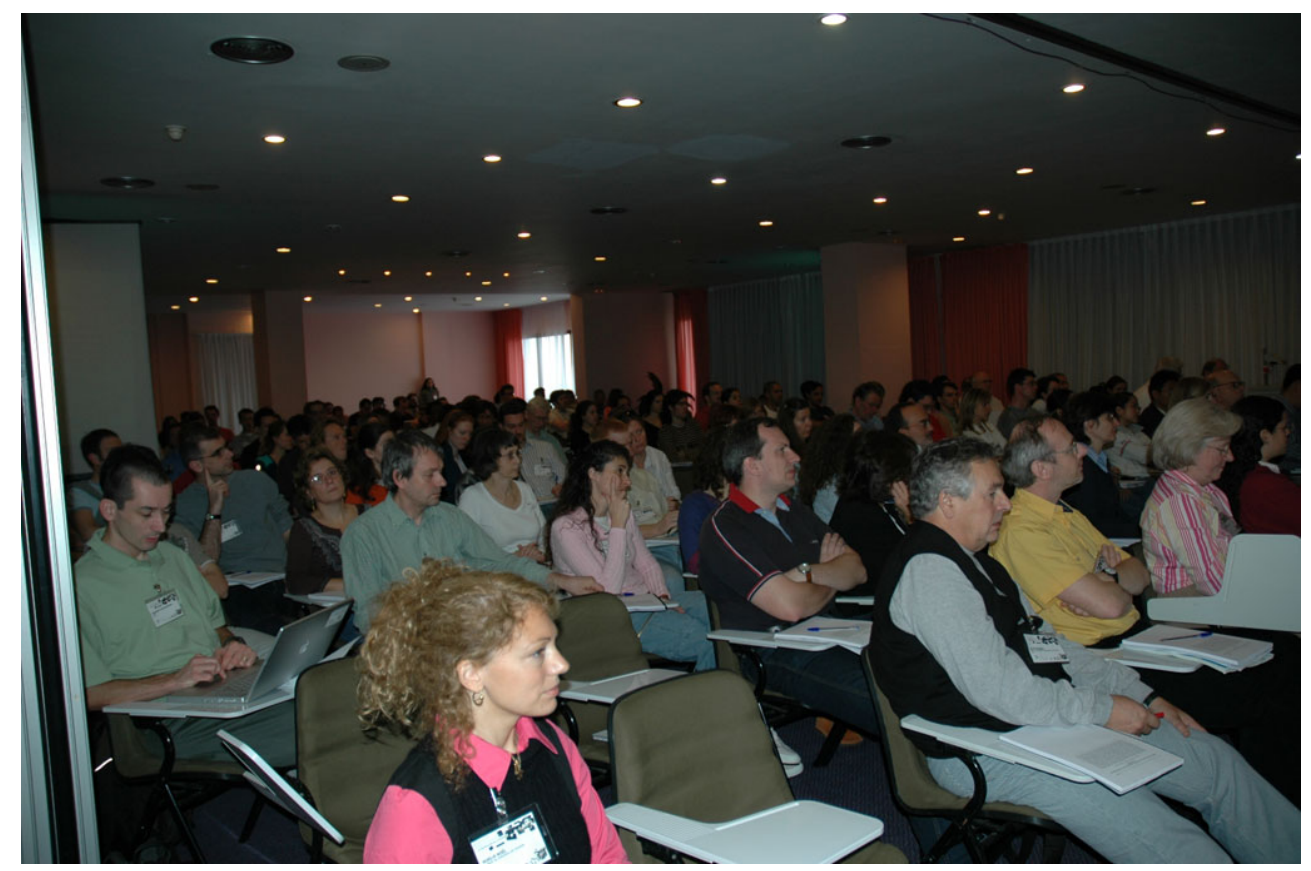

From right to left: Sally Heap, Georges Meynet, Alvio Renzini, Noelia Noël. 